\title{
EFFECTIVE COMPUTATIONS OF THE ATIYAH-BOTT FORMULA
}

\author{
GIOSUE MURATORE AND CSABA SCHNEIDER
}

\begin{abstract}
We present an implementation of the Atiyah-Bott residue formula for $\bar{M}_{0, m}\left(\mathbb{P}^{n}, d\right)$. We use this implementation to compute a large number of Gromov-Witten invariants of genus 0, including intersection numbers of rational curves on general complete intersections. We also compute some numbers of rational contact curves satisfying suitable Schubert conditions. Our computations confirm known predictions made by Mirror Symmetry. The code we developed for these problems is publicly available and can also be used for other types of computations.
\end{abstract}

\section{INTRODUCTION}

The solution of an enumerative problem in algebraic geometry is often given as the degree of a polynomial of Chern classes of bundles over some moduli space $M$. The computation of such classes is easier when the moduli space and the bundle carry an action of a group $G$. Moreover, the $G$-action on $M$ lifts equivariantly to the total space of many natural bundles (such as the tangent bundle).

Suppose that the fixed locus of the action on $M$ is a finite union of points $\{V\}_{V \in I}$, and $i: V \hookrightarrow M$ is the inclusion. The Atiyah-Bott Theorem (see [Bot67, AB84]) says that the localized top Chern class of the normal bundle $c_{\text {top }}\left(N_{V / M}\right)$ is invertible along the fixed locus of the group action. Thus, for any equivariant class $P$ of $M$, we have the celebrated formula:

$$
\int_{M} P=\sum_{V \in I} \int_{V}^{G} \frac{i^{*}(P)}{c_{\mathrm{top}}\left(N_{V / M}\right)}
$$

The symbol $\int_{V}^{G}$ denotes the $G$-equivariant integral at $V$. The terms in the sum are often easier to compute than the degree on the left-hand side. The first application of this formula for enumerative problems is due to ES96. The results in ES96] include complete lists of numbers of twisted cubic curves and elliptic quartic curves contained in a general complete intersection satisfying suitable Schubert conditions. Kontsevich Kon95 used an analogous formula for the moduli of stable maps $\bar{M}_{0, m}\left(\mathbb{P}^{n}, d\right)$, for arbitrary $n, m$ and $d$. He successfully computed the number of rational quartics contained in a general quintic threefold, confirming the prediction of Mirror Symmetry. His version of the formula is

$$
\int_{\bar{M}_{0, m}\left(\mathbb{P}^{n}, d\right)} P=\sum_{\Gamma} \frac{1}{a_{\Gamma}} \frac{P^{T}(\Gamma)}{c_{\mathrm{top}}^{T}\left(N_{\Gamma}\right)(\Gamma)}
$$

Date: February 15, 2022.

2020 Mathematics Subject Classification. 14N10, 14Q25, 14N35, 14C05, 14-04, 53D12.

Key words and phrases. Legendrian, contact, torus action, Bott formula, enumeration, SageMath, Julia programming language. 
where the right-hand side is a finite sum of rational expressions over a set of combinatorial data (see Section 3). This approach was later generalized to positive genus by GP99. Such results are well known to experts in enumerative geometry.

One of the ingredients of Kontsevich's method is parameterizing the fixed locus of the torus action on $\bar{M}_{0, m}\left(\mathbb{P}^{n}, d\right)$ by graphs colored by the fixed points of the action on $\mathbb{P}^{n}$. All such graphs can be obtained in a few seconds by modern computers, even though their number grows very fast. Any intersection of Chern classes of equivariant vector bundles will be a complicated sum of rational expressions taken over the set of all such graphs. At the very end, however, this sum magically collapses to a rational number, as expected.

The authors implemented computer code to apply the Atiyah-Bott formula to $\bar{M}_{0, m}\left(\mathbb{P}^{n}, d\right)$. The package can be used to to perform a wide variety of computations as long as the equivariant class is given with an explicit formula. Such equivariant classes can be easily implemented inside the package, and so our code can perform analogous computations not described here. Finally, we found classical (and less classical) numbers of curves with geometric conditions. In particular, we found a complete list of numbers of contact curves (that is, the integral curves of a variety with contact structure) meeting arbitrary linear subspaces. The enumeration of such curves, started by [LV11], was only partially known before. Our main result is thus the following theorem.

Theorem 1.1. The numbers of rational contact curves in $\mathbb{P}^{3}$ up to degree 8 meeting arbitrary linear subspaces are given in Table 5.2.

This list includes contact curves meeting an appropriate number of lines in $\mathbb{P}^{3}$, as described in OEIS, A328553].

In Section 2 we review the notation and recall some basic results. In Sections 3 and 4 we briefly describe the Atiyah-Bott formula and our algorithm. In the last section, we compute several Gromov-Witten invariants, including the virtual numbers of rational curves of the quintic threefold, up to degree 9. Some of these numbers were never computed before using the Atiyah-Bott formula. Hiep [Hie16] computed them up to degree 6 (but it is not clear, for us, how his code can be used for computations that are different from those explicitly given in the article). In the same section there are many other applications of our code, including the number of curves with tangency conditions. Our code is written in the Julia programming language [BEKS17] and is freely available.

Acknowledgments. The first author thanks Rahul Pandharipande, Andrea Ricolfi, Xiaowen $\mathrm{Hu}$ and Jieao Song for their help, and Alfredo Donno and Matteo Cavaleri for a fruitful discussion. He was also supported by a postdoctoral fellowship PNPD-CAPES. The second author acknowledges the support of the CNPq projects Produtividade em Pesquisa (project no.: 308212/2019-3) and Universal (project no.: 421624/2018-3). We both are grateful to Israel Vainsencher for his continuous support, and to the referee for their careful reading.

\section{StABle MAPS}

2.1. Moduli space and invariants. A marked stable map to $\mathbb{P}^{n}$ of degree $d$ is a tuple $\left(C, f, p_{1}, \ldots, p_{m}\right)$ in which the components are as follows.

- $C$ is a projective, connected, reduced, at-worst-nodal complex curve of arithmetic genus zero. 
- $f: C \rightarrow \mathbb{P}^{n}$ is a morphism such that $f_{*}[C]$ is a cycle of degree $d$.

- $p_{1}, \ldots, p_{m}$ are regular distinct points of $C$, called marked points.

- If $E \subset C$ is a component contracted by $f$, then $E$ contains at least three points among the marks and nodes of $C$.

The coarse moduli space of such stable maps is denoted by $\bar{M}_{0, m}\left(\mathbb{P}^{n}, d\right)$. It is irreducible of dimension $n+(n+1) d+m-3$, see [FP97.

In this section we will use the following notation.

- $\mathrm{ev}_{i}: \bar{M}_{0, m}\left(\mathbb{P}^{n}, d\right) \rightarrow \mathbb{P}^{n}$ is the evaluation map at the $i^{\text {th }}$ marked point.

- $\delta: \bar{M}_{0, m+1}\left(\mathbb{P}^{n}, d\right) \rightarrow \bar{M}_{0, m}\left(\mathbb{P}^{n}, d\right)$ is the forgetful map of the last point.

Let $h$ be the cohomology class of a hyperplane of $\mathbb{P}^{n}$, and let $a_{1}, \ldots, a_{m}$ be positive integers, such that $\sum_{i=1}^{m} a_{i}=\operatorname{dim} \bar{M}_{0, m}\left(\mathbb{P}^{n}, d\right)$. The following GromovWitten invariant counts the number of curves in $\mathbb{P}^{n}$ of degree $d$ meeting $m$ linear subspaces of codimension $a_{i}$ in general position for $i=1, \ldots, m$ [FP97, Lemma 14]:

$$
\int_{\bar{M}_{0, m}\left(\mathbb{P}^{n}, d\right)} \mathrm{ev}_{1}^{*}\left(h^{a_{1}}\right) \cdots \mathrm{ev}_{m}^{*}\left(h^{a_{m}}\right) .
$$

More generally, we may consider the descendant invariants given as

$$
\int_{\bar{M}_{0, m}\left(\mathbb{P}^{n}, d\right)} \mathrm{ev}_{1}^{*}\left(h^{a_{1}}\right) \cdots \mathrm{ev}_{m}^{*}\left(h^{a_{m}}\right) \cdot \psi_{1}^{a_{1}^{\prime}} \cdots \psi_{m}^{a_{m}^{\prime}},
$$

where $\psi_{i}$ is the first Chern class of the line bundle $\mathbb{L}_{i}$ whose fiber at a moduli point is the cotangent space of the curve at the $i^{\text {th }}$ marked point.

Remark 2.1. Using the projection formula, it is not difficult to see that (2.1) is equal to

$$
\int_{\bar{M}_{0,0}\left(\mathbb{P}^{n}, d\right)} \delta_{*}\left(\operatorname{ev}_{1}^{*}\left(h^{a_{1}}\right)\right) \cdots \delta_{*}\left(\mathrm{ev}_{1}^{*}\left(h^{a_{m}}\right)\right) .
$$

In the next subsection we will see that by reducing the number of marks we will speed up the computations for the Atiyah-Bott formula. Indeed, by Mur20, Theorem 4.3], $\delta_{*}\left(h^{a_{1}}\right)$ is a polynomial in the Chern classes of equivariant vector bundles; see [ES96, Proposition 3.4] for a similar discussion.

Remark 2.2. Enumerative problems of curves with tangency conditions often involve the bundles $\mathrm{J}^{p}\left(\mathrm{ev}_{i}^{*} \mathcal{O}_{\mathbb{P}^{n}}(z)\right)$ of $p$-jets (see [EH16, Section 7.2] for the definition). The Euler class of this bundle can be obtained by recursion using the condition $\mathrm{J}^{0}\left(\mathrm{ev}_{i}^{*} \mathcal{O}_{\mathbb{P}^{n}}(z)\right):=\mathrm{ev}_{i}^{*} \mathcal{O}_{\mathbb{P}^{n}}(z)$ and by the exact sequence

$$
0 \rightarrow \mathrm{ev}_{i}^{*} \mathcal{O}_{\mathbb{P}^{n}}(z) \otimes \mathbb{L}_{i}^{\otimes p} \rightarrow \mathrm{J}^{p}\left(\mathrm{ev}_{i}^{*} \mathcal{O}_{\mathbb{P}^{n}}(z)\right) \rightarrow \mathrm{J}^{p-1}\left(\mathrm{ev}_{i}^{*} \mathcal{O}_{\mathbb{P}^{n}}(z)\right) \rightarrow 0 .
$$

Let $X \stackrel{i}{\hookrightarrow} \mathbb{P}^{n}$ be a general complete intersection of multidegree $b=\left(b_{1}, \ldots, b_{s}\right)$, such that $n+1 \geq \sum b_{i}$. That is, $X$ is the zero locus of a general section $\sigma$ of the vector bundle $F=\bigoplus_{i=1}^{s} \mathcal{O}_{\mathbb{P}^{n}}\left(b_{i}\right)$. By standard base change theory, $\delta_{*}\left(\mathrm{ev}_{m+1}^{*} F\right)$ is a vector bundle on $\bar{M}_{0, m}\left(\mathbb{P}^{n}, d\right)$ of rank $\sum\left(b_{i} d+1\right)=s+\sum b_{i} d$. The section defining $X$ induces in a natural way a section $\sigma^{\prime}$ of $\delta_{*}\left(\mathrm{ev}_{m+1}^{*} F\right)$ whose zero locus is the locus in $\bar{M}_{0, m}\left(\mathbb{P}^{n}, d\right)$ of maps with image contained in $X$. In other words, the section $\sigma^{\prime}$ vanishes at a moduli point of a stable map $f: C \rightarrow \mathbb{P}^{n}$ if and only if $f=i \circ f^{\prime}$ for some map $f^{\prime}: C \rightarrow X$, and $i_{*} f_{*}[C]$ has degree $d$. 
We may consider the Gromov-Witten invariant

$$
\int_{\bar{M}_{0, m}\left(\mathbb{P}^{n}, d\right)} \operatorname{ev}_{1}^{*}\left(h^{a_{1}}\right) \cdots \mathrm{ev}_{m}^{*}\left(h^{a_{m}}\right) \cdot c_{\mathrm{top}}\left(\delta_{*}\left(\mathrm{ev}_{m+1}^{*} F\right)\right) .
$$

This invariant counts the virtual number of rational curves 11 in $X$ meeting general hyperplanes of codimension $a_{i}$ for $i=1, \ldots, m$.

Remark 2.3. Equation (2.4) does not coincide with the number of rational curves in $X$ meeting general hyperplanes of codimension $a_{i}$ for $i=1, \ldots, m$ if we have contribution, for example, from stable maps with automorphisms. That is, (2.4) is not enumerative. The Aspinwall-Morrison formula says that if $X$ is a CalabiYau threefold and $d=k_{1} k_{2}$, then the contribution to (2.4) from smooth rational curves of degree $k_{1}$ is $k_{2}^{-3}$, see [AM93, Voi96. Manin Man95 computed such a contribution by proving that:

$$
\int_{\bar{M}_{0,0}\left(\mathbb{P}^{1}, d\right)} c_{\mathrm{top}}\left(R^{1} \delta_{*} \mathrm{ev}_{1}^{*}\left(\mathcal{O}_{\mathbb{P}^{1}}(-1)\right)^{\oplus 2}\right)=\frac{1}{d^{3}} .
$$

When $X$ is Fano and convex, then it is also homogeneous [Pan13]. In that case, we can apply [Kle74, 2 Theorem] to make the intersection in (2.4) be transverse, so (2.4) is enumerative.

Let $n$ be odd. It is well-known that $\mathbb{P}^{n}$ has a unique contact structure 2 given by the line subbundle $\mathcal{O}_{\mathbb{P}^{n}}(-2) \hookrightarrow \Omega_{\mathbb{P}^{n}}$, see [Kob59. The curves pointwise tangent to the contact distribution are particularly interesting in the classification of contact structures (see, e.g., [Ye94]). Such curves are called contact.

Definition 2.4. A rational curve $C \subset \mathbb{P}^{n}$ of degree $d$ is contact if $s_{\mid T C} \equiv 0$ for every local section $s$ of $\mathcal{O}_{\mathbb{P}^{n}}(-2)$.

Let $\omega_{\delta}$ be the relative dualizing sheaf of $\delta: \bar{M}_{0, m+1}\left(\mathbb{P}^{n}, d\right) \rightarrow \bar{M}_{0, m}\left(\mathbb{P}^{n}, d\right)$. We may define the equivariant vector bundle over $\bar{M}_{0, m}\left(\mathbb{P}^{n}, d\right)$

$$
\mathcal{E}_{d, m}:=\delta_{*}\left(\omega_{\delta} \otimes \mathrm{ev}_{m+1}^{*} \mathcal{O}_{\mathbb{P}^{n}}(2)\right) .
$$

The first author proved in Mur20 that the number of rational contact curves of degree $d$ meeting general linear hyperplanes of codimension $a_{i}$ for $i=1, \ldots, m$ is

$$
\int_{\bar{M}_{0, m}\left(\mathbb{P}^{n}, d\right)} \operatorname{ev}_{1}^{*}\left(h^{a_{1}}\right) \cdots \mathrm{ev}_{m}^{*}\left(h^{a_{m}}\right) \cdot c_{\mathrm{top}}\left(\mathcal{E}_{d, m}\right) .
$$

We will be interested in rational contact space curves of degree $d$ meeting $a$ general points and $b$ general lines, where $a+2 b=2 d+1$.

Remark 2.5. Both (2.4) and (2.6) can be reduced to the case when $m=0$, thanks to Remark 2.1, which holds in these cases as well.

\footnotetext{
${ }^{1}$ That is, the number of stable maps with multiplicity.

${ }^{2} \mathrm{~A}$ contact structure on a smooth complex variety $X$ of dimension $2 n+1$ is a line subbundle $L \hookrightarrow \Omega_{X}$ such that for every local section $s$ of $L$, the section $s \wedge(\mathrm{d} s)^{\wedge n}$ is nowhere zero.
} 


\section{LocAlizAtion}

In this section we will briefly recall the powerful method of localization, following Kon95 and CK99. Let $T=\left(\mathbb{C}^{*}\right)^{n+1}$ be the torus acting on $\mathbb{P}^{n}$ with the standard action, and let $\left\{x_{i}\right\}_{i=0}^{n}$ be the fixed points. The action of the torus $T$ on $\mathbb{P}^{n}$ lifts to $\bar{M}_{0, m}\left(\mathbb{P}^{n}, d\right)$ by composition. That is, if $f: C \rightarrow \mathbb{P}^{n}$ is a stable map, for every $t \in T$ we have a new stable map $t \cdot f: C \rightarrow \mathbb{P}^{n}$. The fixed locus of this action is usually denoted by $\bar{M}_{0, m}\left(\mathbb{P}^{n}, d\right)^{T}$, its connected components are finite and naturally labelled by isomorphism classes of combinatorial data $\Gamma=(g, \mathbf{c}, \mathbf{w}, \mathbf{q})$, called decorated graphs defined as follows.

(1) $g$ is a tree; that is $g$ is a simple undirected, connected graph without cycles. We denote by $V_{\Gamma}$ and $E_{\Gamma}$ the sets of vertices and edges of $g$.

(2) The vertices of $g$ are colored by the points $\left\{x_{i}\right\}_{i=0}^{n}$; the coloring is given by a map $\mathbf{c}: V_{\Gamma} \rightarrow\left\{x_{0}, \ldots, x_{n}\right\}$ such that $\mathbf{c}(v) \neq \mathbf{c}(w)$ if $v$ and $w$ are two vertices in the same edge.

(3) Each edge of $g$ is weighted by a map w: $E_{\Gamma} \rightarrow \mathbb{Z}$, such that for every $e \in E_{\Gamma}, \mathbf{w}(e)>0$ and $\sum_{e \in E_{\Gamma}} \mathbf{w}(e)=d$.

(4) The marks of the vertices of $g$ are given by a map q: $A \rightarrow V_{\Gamma}$, where $A=\{1, \ldots, m\}$ if $m>0$, and $A=\emptyset$ if $m=0$.

In other words, the number of all connected components of $\bar{M}_{0, m}\left(\mathbb{P}^{n}, d\right)^{T}$ equals the number of possible tuples $\Gamma=(g, \mathbf{c}, \mathbf{w}, \mathbf{q})$ counted modulo isomorphism. An isomorphism between two such tuples is an isomorphism of graphs preserving $\mathbf{c}, \mathbf{w}$ and $\mathbf{q}$.

Example 3.1. Consider the space $\bar{M}_{0,0}\left(\mathbb{P}^{2}, 1\right)$. Since the degree is 1 , all possible graphs must have one edge by item (3) and the weight of this edge will be 1 . We can use three colors, namely $x_{0}, x_{1}, x_{2}$, and so we have just three possible coloring (modulo isomorphism). Item (4) is an empty condition since $m=0$. Hence, we have three connected components labelled by the following three graphs.

$$
x_{0} \stackrel{1}{-} x_{1} \quad x_{0} \stackrel{1}{-} x_{2} \quad x_{1} \stackrel{1}{-} x_{2}
$$

Example 3.2. For the space $\bar{M}_{0,2}\left(\mathbb{P}^{1}, 1\right)$, the number of connected components is four, labelled by the following graphs:
$\{1,2\}$
$x_{0} \frac{1}{-} x_{1}$
$\begin{array}{lll}\{1\} & & \{2\} \\ x_{0} & 1 & x_{1}\end{array}$
$\begin{array}{lrr}\{2\} & \{1\} \\ x_{0} & 1 & x_{1}\end{array}$
$x_{0} \stackrel{1}{\quad\{1,2\}}$

The numbers inside the brackets are the marks of the vertices.

Example 3.3. For the space $\bar{M}_{0,0}\left(\mathbb{P}^{n}, 2\right)$, the graphs may have one or two edges by item (3). It can easily be seen that we have the following possibilities.

$$
\begin{array}{rl}
x_{i} \frac{2}{x_{j}} & 0 \leq i<j \leq n \\
x_{i} \stackrel{1}{1} x_{j} \frac{1}{} x_{k} & 0 \leq i<k \leq n \\
& 0 \leq j \leq n, j \neq i, k
\end{array}
$$

The correspondence between decorated graphs and connected components of $\bar{M}_{0, m}\left(\mathbb{P}^{n}, d\right)^{T}$ can be made explicit in the following way. For each decorated graph $\Gamma=(g, \mathbf{c}, \mathbf{w}, \mathbf{q})$, we associate the substack $\overline{\mathcal{M}}_{\Gamma}$ of all marked stable maps $\left(C, f, p_{1}, \ldots, p_{m}\right) \in \bar{M}_{0, m}\left(\mathbb{P}^{n}, d\right)$ such that the followings hold. 
(1) There is a bijective correspondence between vertices $v$ of $g$ and connected components $C_{v}$ of $f^{-1}\left\{x_{0}, \ldots, x_{n}\right\}$. In particular, $C_{v}$ is either a point or a contracted union of curves. The color of $v$ is the image of $C_{v}$.

(2) There is a bijective correspondence between edges $e=(v, w)$ of $g$ and irreducible components of $C$ mapped to the line through $\mathbf{c}(v)$ and $\mathbf{c}(w)$, with a map of degree $\mathbf{w}(e)$.

(3) If $m>0$, the set of marked points $\left\{p_{i}\right\}_{i=1}^{m}$ is mapped to the set of fixed points $\left\{x_{i}\right\}_{i=0}^{n}$. We define a map $\mathbf{q}:\{1, \ldots, m\} \rightarrow V_{\Gamma}$ such that $\mathbf{q}(i)=v$ if and only if $p_{i} \in C_{v}$.

Conversely, every connected component of the fixed locus is of the form $\overline{\mathcal{M}}_{\Gamma}$ for some decorated graph $\Gamma$.

We denote by $\operatorname{val}(v)$ the number of edges connected to the vertex $v$. Note that $C_{v}$ is of dimension 1 if and only if $n(v):=\operatorname{val}(v)+\left|\mathbf{q}^{-1}(v)\right|$ satisfies $n(v) \geq 3$. Let us denote

$$
M_{\Gamma}:=\prod_{v: \operatorname{dim} C_{v}=1} \bar{M}_{0, n(v)} .
$$

If $|\operatorname{Aut}(\Gamma)|$ is the order of the group of automorphisms of the decorated graph $\Gamma$, there exists a group of order

$$
a_{\Gamma}:=|\operatorname{Aut}(\Gamma)| \cdot \prod_{e \in E_{g}} \mathbf{w}(e)
$$

acting on $M_{\Gamma}$ with quotient map given by:

$$
\phi: M_{\Gamma} \rightarrow \overline{\mathcal{M}}_{\Gamma} .
$$

For any space $X$ with a $T$-action, let us denote by $H_{T}^{*}(X)$ the equivariant cohomology of $X$. Moreover, let us denote by $\lambda_{i}$ the weight of the $T$-action on $\mathcal{O}_{\mathbb{P} n}(-1)_{\mid x_{i}}$. Hence the equivariant cohomology of a single point is isomorphic to

$$
H_{T}^{*}(\operatorname{Spec}(\mathbb{C})) \cong \mathbb{C}\left[\lambda_{0}, \ldots, \lambda_{n}\right]
$$

Let us denote by $\mathcal{R}_{T}$ the field of fractions of $H_{T}^{*}(\operatorname{Spec}(\mathbb{C}))$. By [CK99, Proposition 9.1.2] we know that there is an isomorphism

$$
H_{T}^{*}\left(\bar{M}_{0, m}\left(\mathbb{P}^{n}, d\right)\right) \otimes \mathcal{R}_{T} \cong \bigoplus_{\Gamma} H_{T}^{*}\left(\overline{\mathcal{M}}_{\Gamma}\right) \otimes \mathcal{R}_{T} .
$$

From this isomorphism we deduce the Atiyah-Bott formula

$$
\int_{\bar{M}_{0, m}\left(\mathbb{P}^{n}, d\right)} P=\sum_{\Gamma} \frac{1}{a_{\Gamma}} \frac{P^{T}(\Gamma)}{c_{\mathrm{top}}^{T}\left(N_{\Gamma}\right)(\Gamma)},
$$

where

- $N_{\Gamma}$ is the normal bundle (as a stack) of $\overline{\mathcal{M}}_{\Gamma}$;

- $P$ is a polynomial in Chern classes of equivariant vector bundles of the moduli space $\bar{M}_{0, m}\left(\mathbb{P}^{n}, d\right)$;

- $P^{T}$ is the equivariant polynomial of $P$, obtained by substitution of the Chern classes appearing in $P$ with the corresponding equivariant Chern classes;

- $c_{\text {top }}^{T}\left(N_{\Gamma}\right)$ is the equivariant top Chern class of $N_{\Gamma}$, i.e., same as before when $P=c_{\text {top }}\left(N_{\Gamma}\right)$

- $P^{T}(\Gamma)$ is the restriction to $H_{T}^{*}\left(\overline{\mathcal{M}}_{\Gamma}\right)$ of $P^{T}$. 
In other words, by Equation 3.2, we can compute every integral of $P$ as a sum of elements of $\mathcal{R}_{T}$.

3.1. Explicit formulas. We use the following notation.

- For each $v \in V_{\Gamma}$ colored by $x_{i}$, the symbols $\lambda_{v}$ and $\lambda_{x_{i}}$ denote $\lambda_{i}$.

- For each $e \in E_{\Gamma}$, the symbols $d_{e}$ and $\left(e_{1}, e_{2}\right)$ are, respectively, the weight of $e$ and the pair of colors of its vertices.

- For each $v \in V_{\Gamma}$, a flag $F$ of $v$ is a pair $(v, e)$ where $e$ is an edge having $v$ as one of its vertices. Moreover if $w$ is the other vertex of $e$, we denote by $\omega_{F}:=\left(\lambda_{v}-\lambda_{w}\right) / d_{e}$, and by $F_{v}$ the set of flags of $v$.

All localizations of the invariants in Section 2 can be explicitly computed. See [Kon95, CK99, Mur20] and reference therein for the following equivariant classes (here $b>0>k$ ):

$$
\begin{aligned}
c_{\mathrm{top}}^{T}\left(\delta_{*} \mathrm{ev}_{m+1}^{*}\left(\mathcal{O}_{\mathbb{P}^{n}}(b)\right)\right)(\Gamma) & =\prod_{e \in E_{\Gamma}} \prod_{\alpha=0}^{b d_{e}} \frac{\alpha \lambda_{e_{1}}+\left(b d_{e}-\alpha\right) \lambda_{e_{2}}}{d_{e}} \prod_{v \in V_{\Gamma}}\left(b \lambda_{v}\right)^{1-\operatorname{val}(v) ;} \\
c_{\mathrm{top}}^{T}\left(\mathcal{E}_{d, m}\right)(\Gamma) & =\prod_{e \in E_{\Gamma}} \prod_{\alpha=1}^{2 d_{e}-1} \frac{\alpha \lambda_{e_{1}}+\left(2 d_{e}-\alpha\right) \lambda_{e_{2}}}{d_{e}} \prod_{v \in V_{\Gamma}}\left(2 \lambda_{v}\right)^{\operatorname{val}(v)-1} ; \\
c_{\mathrm{top}}^{T}\left(R^{1} \delta_{*} \mathrm{ev}_{m+1}^{*}\left(\mathcal{O}_{\mathbb{P}^{n}}(k)\right)\right)(\Gamma) & =\prod_{e \in E_{\Gamma}} \prod_{\alpha=-1}^{k d_{e}-1} \frac{\alpha \lambda_{e_{1}}+\left(k d_{e}-\alpha\right) \lambda_{e_{2}}}{d_{e}} \prod_{v \in V_{\Gamma}}\left(k \lambda_{v}\right)^{\operatorname{val}(v)-1} ; \\
(3.3) \quad\left[\delta_{*}\left(\mathrm{ev}_{m+1}^{*} h^{r+1}\right)\right]^{T}(\Gamma) & =\sum_{e \in E_{\Gamma}} \sum_{t=0}^{r} d_{e} \lambda_{e_{1}}^{t} \lambda_{e_{2}}^{r-t} ; \\
(3.4) \quad \operatorname{ev}_{j}^{*}\left(\mathcal{O}_{\mathbb{P}^{n}}(1)\right) & =\lambda_{\mathbf{q}(j)} .
\end{aligned}
$$

In order to compute the contribution from the Euler class of $N_{\Gamma}$ and from the $\psi$-classes, we introduce the following notation (see Kon95, 3.3.4])

$$
\begin{array}{r}
X(\Gamma):=\prod_{e \in E_{\Gamma}}\left(\frac{(-1)^{d_{e}}}{\left(d_{e} !\right)^{2}}\left(\frac{d_{e}}{\lambda_{e_{1}}-\lambda_{e_{2}}}\right)^{2 d_{e}} \prod_{k \neq e_{1}, e_{2}} \prod_{a=0}^{d_{e}} \frac{d_{e}}{a \lambda_{e_{1}}+\left(d_{e}-a\right) \lambda_{e_{2}}-d_{e} \lambda_{k}}\right) \\
\times \prod_{v \in V_{\Gamma}}\left(\prod_{j \neq \mathbf{c}(v)} \lambda_{v}-\lambda_{j}\right)^{\operatorname{val}(v)-1} .
\end{array}
$$

Let $\left\{a_{i}\right\}_{i=1}^{m}$ be non-negative integers, and let $v$ be a vertex. Let $S_{v}:=\mathbf{q}^{-1}(v)=$ $\left\{j_{1}, \ldots, j_{k}\right\}$ denote the set of marks mapped to $v$, and let $\overline{S_{v}}:=\sum_{i \in S_{v}} a_{i}$ and $N:=n(v)-3-\overline{S_{v}}$. If $n(v) \geq 3$ and $N \geq 0$, we recall the multinomial coefficients

$$
\left(\begin{array}{c}
n(v)-3 \\
N, a_{j_{1}}, \ldots, a_{j_{k}}
\end{array}\right)=\frac{(n(v)-3) !}{N ! \cdot a_{j_{1}} ! \cdots a_{j_{k}} !} .
$$

We may extend the value of equation (3.5) in the following way:

- if $\overline{S_{v}}>n(v)-3 \geq 0$, then its value is 0 ;

- if $S_{v}=\emptyset$ or $\overline{S_{v}}=0$, then its value is 1 ;

- if $S_{v}=\left\{a_{j_{1}}\right\}$ and $n(v)=2$, then its value is $(-1)^{a_{j_{1}}}$.

As we saw in (3.1), $M_{\Gamma}$ is a product of moduli spaces $\bar{M}_{0, n(v)}$ when $n(v) \geq 3$. In each space, we have formal classes $\psi_{F}$ for each $F \in F_{v}$, and $\psi_{i}$ for each $i \in S_{v}$. 
Lemma 3.4. We have the following integral in formal variables:

$$
\int_{\bar{M}_{0, n(v)}} \frac{\prod_{i \in S_{v}} \psi_{i}^{a_{i}}}{\prod_{F \in F_{v}} \omega_{F}-\psi_{F}}=\left(\begin{array}{c}
n(v)-3 \\
N, a_{j_{1}}, \ldots, a_{j_{k}}
\end{array}\right) \prod_{F \in F_{v}} \omega_{F}^{-1}\left(\sum_{F \in F_{v}} \omega_{F}^{-1}\right)^{N} .
$$

Proof. First we compute that

$$
\begin{aligned}
\int_{\bar{M}_{0, n(v)}} \frac{\prod_{i \in S_{v}} \psi_{i}^{a_{i}}}{\prod_{F \in F_{v}} \omega_{F}-\psi_{F}} & =\int_{\bar{M}_{0, n(v)}} \frac{\prod_{i \in S_{v}} \psi_{i}^{a_{i}}}{\prod_{F \in F_{v}} \omega_{F}\left(1-\frac{\psi_{F}}{\omega_{F}}\right)} \\
& =\prod_{F \in F_{v}} \omega_{F}^{-1} \int_{\bar{M}_{0, n(v)}} \frac{\prod_{i \in S_{v}} \psi_{i}^{a_{i}}}{\prod_{F \in F_{v}}\left(1-\frac{\psi_{F}}{\omega_{F}}\right)} \\
& =\prod_{F \in F_{v}} \omega_{F}^{-1} \sum_{K} \int_{\bar{M}_{0, n(v)}} \prod_{i \in S_{v}} \prod_{F \in F_{v}} \psi_{i}^{a_{i}}\left(\frac{\psi_{F}}{\omega_{F}}\right)^{k_{F}}
\end{aligned}
$$

where the sum runs over all partition $3=\left\{k_{F}\right\}_{F \in F_{v}}$ of $N$. Note that we used $1 /(1-x)=1+x+x^{2}+\ldots$ and the fact that the integral is zero if the sum of the exponents of all $\psi$-classes is not $n(v)-3$. Finally

$$
\begin{aligned}
\sum_{K}\left(\prod_{F \in F_{v}} \frac{1}{\omega_{F}^{k_{F}}}\right) \int_{\bar{M}_{0, n(v)}} \prod_{i \in S_{v}} \psi_{i}^{a_{i}} \prod_{F \in F_{v}} \psi_{F}^{k_{F}} & =\sum_{K}\left(\prod_{F \in F_{v}} \frac{1}{\omega_{F}^{k_{F}}}\right) \frac{(n(v)-3) !}{\prod_{i \in S_{v}} a_{i} ! \prod_{F} k_{F} !} \\
& =\frac{(n(v)-3) !}{N ! \prod_{i \in S_{v}} a_{i} !} \sum_{K} N ! \prod_{F \in F_{v}} \frac{1}{k_{F} ! \omega_{F}^{k_{F}}} \\
& =\left(\begin{array}{c}
n(v)-3 \\
N, a_{j_{1}}, \ldots, a_{j_{k}}
\end{array}\right)\left(\sum_{F \in F_{v}} \frac{1}{\omega_{F}}\right)^{N} .
\end{aligned}
$$

The value of the integral in the first equation follows from [Wit91, Kon92].

In the case $n(v) \leq 2$, we may formally extend the integral according to (3.5):

$$
\begin{aligned}
\int_{\bar{M}_{0,1}} \frac{1}{\omega_{F}-\psi_{F}} & =\omega_{F} \\
\int_{\bar{M}_{0,2}} \frac{\psi_{i}^{a_{j_{1}}}}{\omega_{F}-\psi_{F}} & =\left(-\omega_{F}\right)^{a_{j_{1}}} \\
\int_{\bar{M}_{0,2}} \frac{1}{\prod_{F \in F_{v}} \omega_{F}-\psi_{F}} & =\frac{1}{\sum_{F \in F_{v}} \omega_{F}}\left(\text { if } S_{v}=\emptyset\right) .
\end{aligned}
$$

The contribution from the Euler class of $N_{\Gamma}$ and from the $\psi$-classes is

$$
\frac{\left[\psi_{1}^{a_{1}} \cdots \psi_{m}^{a_{m}}\right]^{T}(\Gamma)}{c_{\mathrm{top}}^{T}\left(N_{\Gamma}\right)(\Gamma)}=X(\Gamma) \prod_{v \in V_{\Gamma}} \int_{\bar{M}_{0, n(v)}} \frac{\prod_{i \in S_{v}} \psi_{i}^{a_{i}}}{\prod_{F \in F_{v}} \omega_{F}-\psi_{F}} .
$$

This is proved in [Kon95, 3.3] when $a_{i}=0$ for $i=1, \ldots, m$ (see subsections 3.3.3 and 3.3.5 for the explicit formulas). For the general case, we refer to [LP04, 3.7] or [LS17, Section 4]. We use Lemma 3.4 to compute the right-hand side of (3.6).

\footnotetext{
${ }^{3}$ A partition of $N$ is an indexed tuple of non-negative integers whose sum is $N$.
} 
In the code, in order to have the $\psi$-classes as an external function independent of $c_{\text {top }}^{T}\left(N_{\Gamma}\right)(\Gamma)$, we implemented the following two functions:

$$
\begin{aligned}
\text { Euler_inv } & :=X(\Gamma) \prod_{v \in V_{\Gamma}} \int_{\bar{M}_{0, n(v)}} \frac{1}{\prod_{F \in F_{v}} \omega_{F}-\psi_{F}} \\
& =X(\Gamma) \prod_{v \in V_{\Gamma}} \prod_{F \in F_{v}} \omega_{F}^{-1}\left(\sum_{F \in F_{v}} \omega_{F}^{-1}\right)^{n(v)-3} \\
\text { Psi } & :=\prod_{v \in V_{\Gamma}} \int_{\bar{M}_{0, n(v)}} \frac{\prod_{i \in S_{v}} \psi_{i}^{a_{i}}}{\prod_{F \in F_{v}} \omega_{F}-\psi_{F}}\left(\int_{\bar{M}_{0, n(v)}} \frac{1}{\prod_{F \in F_{v}} \omega_{F}-\psi_{F}}\right)^{-1} \\
& =\prod_{v \in V_{\Gamma}}\left(\begin{array}{c}
n(v)-3 \\
n(v)-3-\overline{S_{v}}, a_{j_{1}}, \ldots, a_{j_{k}}
\end{array}\right)\left(\sum_{F \in F_{v}} \omega_{F}^{-1}\right)^{-\overline{S_{v}}} .
\end{aligned}
$$

Example 3.5. Suppose we want to compute

$$
I=\int_{\bar{M}_{0,0}\left(\mathbb{P}^{2}, 1\right)} \delta_{*}\left(\mathrm{ev}_{1}^{*}\left(h^{2}\right)\right) \cdot \delta_{*}\left(\mathrm{ev}_{1}^{*}\left(h^{2}\right)\right) .
$$

When $\Gamma$ is

$$
x_{i} \stackrel{1}{-} x_{j} \quad 0 \leq i<j \leq 2,
$$

we know that

$$
\begin{aligned}
{\left[\delta_{*}\left(\mathrm{ev}_{1}^{*} h^{2}\right)\right]^{T}(\Gamma) } & =\lambda_{i}+\lambda_{j} \\
\frac{1}{c_{\mathrm{top}}^{T}\left(N_{\Gamma}\right)(\Gamma)} & =\frac{1}{\lambda_{j}-\lambda_{k}} \frac{1}{\lambda_{i}-\lambda_{k}}
\end{aligned}
$$

where $k \in\{0,1,2\} \backslash\{i, j\}$. By Example 3.2, since $\bar{M}_{0,0}\left(\mathbb{P}^{2}, 1\right)^{T}$ has three connected components, the application of the Atiyah-Bott formula will express $I$ as the sum of three rational polynomials:

$$
I=\frac{\lambda_{0}+\lambda_{1}}{\lambda_{1}-\lambda_{2}} \frac{\lambda_{0}+\lambda_{1}}{\lambda_{0}-\lambda_{2}}+\frac{\lambda_{0}+\lambda_{2}}{\lambda_{2}-\lambda_{1}} \frac{\lambda_{0}+\lambda_{2}}{\lambda_{0}-\lambda_{1}}+\frac{\lambda_{1}+\lambda_{2}}{\lambda_{2}-\lambda_{0}} \frac{\lambda_{1}+\lambda_{2}}{\lambda_{1}-\lambda_{0}}=1
$$

\section{The Algorithm}

Algorithm 1 describes the computation of the equivariant class $\mathrm{P}$ in the moduli space $\bar{M}_{0, m}\left(\mathbb{P}^{n}, d\right)$. The for loop starting at line 3 runs through the isomorphisms classes of tree graphs with at least 2 and at most $d+1$ vertices. The list of such graphs with up to 14 vertices was precomputed using the SageMath computational algebra system and stored in a file as a list of Prüfer sequencest Then the for loop starting from line 4 runs through all the equivalence classes of colorings of the current value of $g$. These colorings are also precomputed with SageMath and stored in a file as a list of arrays of integers. The variable aut in line 5 will hold the size of the automorphisms group of the colored graph $g$ with respect to the coloring $c$. Then the loops starting from lines 6 and 7 run through all possible markings and

\footnotetext{
${ }^{4}$ Given a tree $g$ of $k$ vertices and a bijective map $f: V_{\Gamma} \rightarrow\{1,2, \ldots, k\}$, a Prüfer sequence is a sequence of $k-2$ numbers in $\{1,2, \ldots, k\}$, which recovers $g$ and $f$ modulo automorphisms of $g$.
} 


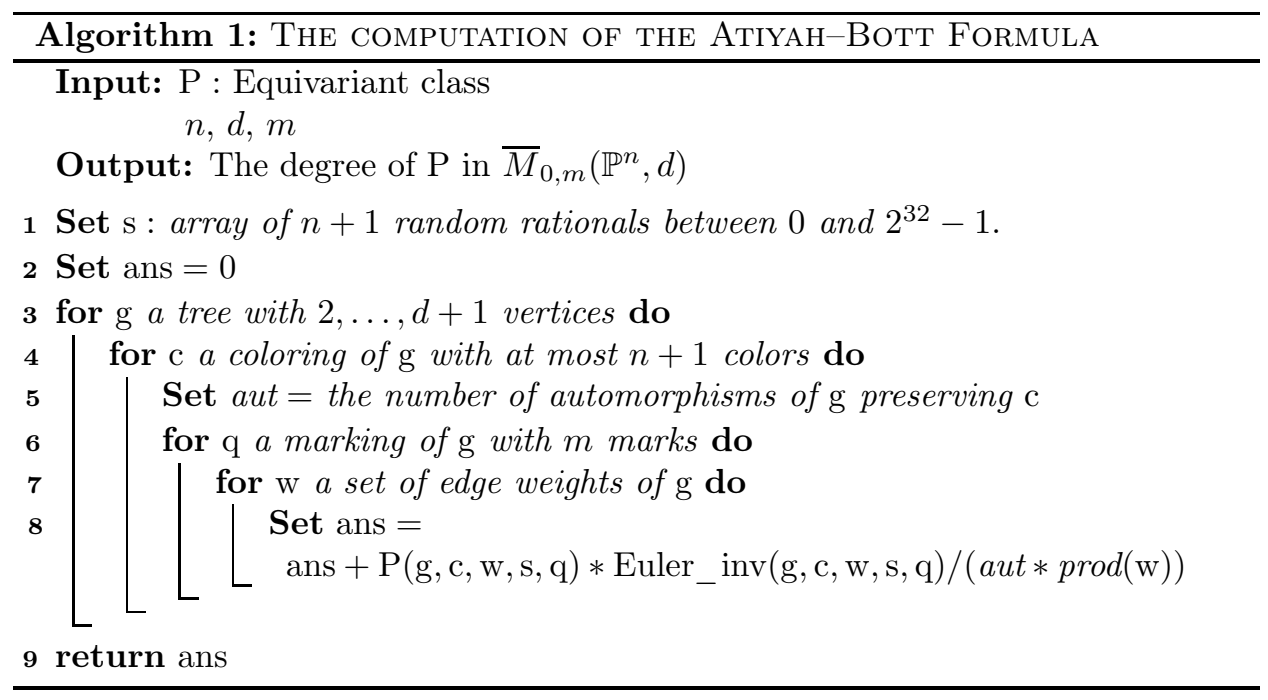

edge weights without considering equivalence. The function $\operatorname{prod}(\mathrm{w})$ denotes the product of all weights.

Our implementation of an equivariant class is a Julia function, returning a rational number, with arguments including $\mathrm{g}, \mathrm{c}, \mathrm{w}, \mathrm{s}, \mathrm{q}$, such that:

- $\mathrm{g}$ is a graph implemented using the Julia package Graphs [FBS ${ }^{+21}$. It is generated from a Prüfer sequence, which gives an enumeration of the vertices of $g$.

- $c$ is an array of integers of length equals to the number of vertices of $g$, the $i^{\text {th }}$ entry of $\mathrm{c}$ is the color of the $i^{\text {th }}$ vertex.

- $w$ is an array of integers of length equals to the number of edges of $g$, whose sum is $d$.

- $\mathbf{s}$ is an array of random rational numbers of length $n+1$, generated at the beginning of the computation.

- $\mathrm{q}$ is an array of integers of length $m$, the $i^{\text {th }}$ entry of $\mathrm{q}$ is the vertex containing the $i^{\text {th }}$ mark.

Not all functions need the argument q, and some functions need additional arguments. The symbol $\lambda_{v}$ is implemented as $\mathrm{s}[\mathrm{c}[\mathrm{v}]]$. Here $\mathrm{v}$ is the number of a vertex, so $\mathrm{c}[\mathrm{v}]$ is the color of that vertex, and finally $\mathrm{s}[\mathrm{c}[\mathrm{v}]]$ is a rational number. On the other hand, $\lambda_{\mathbf{q}(j)}$ is implemented in the obvious way. For example, the equivariant class (3.4) is

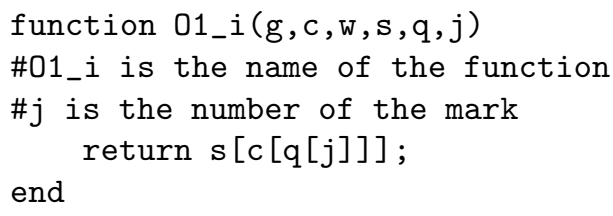

This is the simplest equivariant class that we can define. All other equivariant classes can be defined using the operations of the rational numbers. Those operations are well defined in the entries of the array s. 
For each edge we associate an entry of w using the structure Dictionary provided by the Julia language. The two vertices of an edge $e$ are denoted, in Julia, by $\operatorname{src}(e)$ and $\operatorname{dst}(e)$.

The equivariant class (3.3) is the following (exp is the exponent of $h$ ).

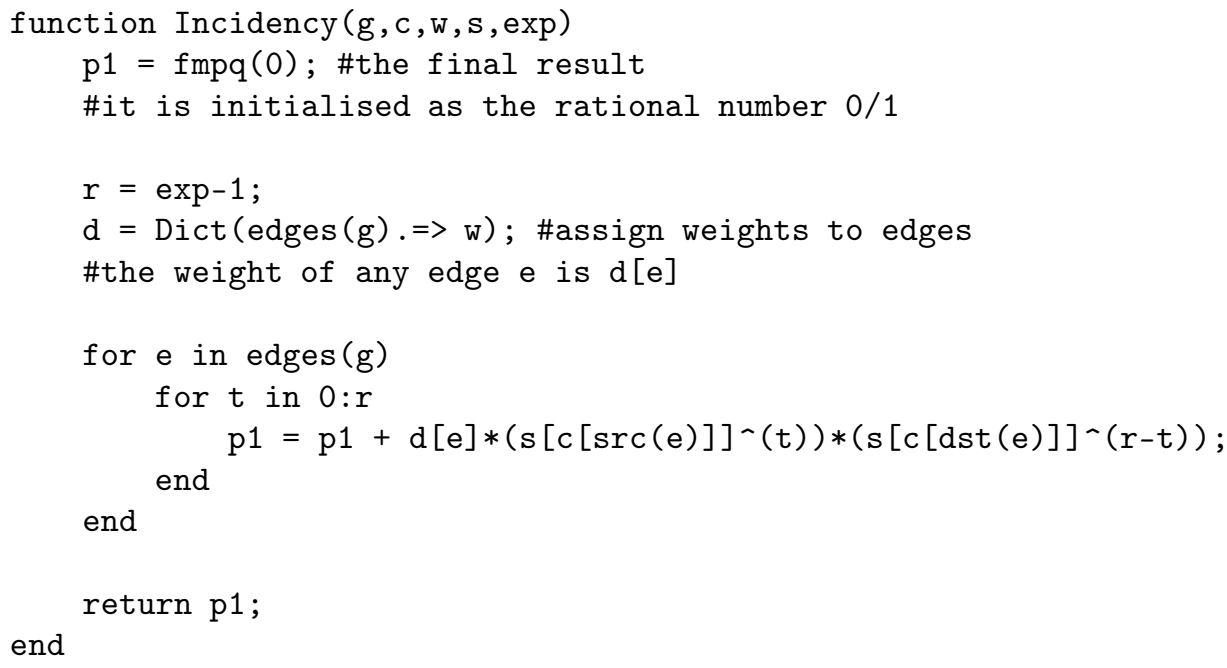

We implemented all equivariant classes listed in Section 3 following the same strategy. This is possible since those classes are written in explicit combinatorial terms. Even if some of them seem complicated, they are relatively easy to implement in Julia.

The function $\mathrm{P}$, defined by the user, is any combination of Julia functions implementing equivariant classes, see the next section for some examples. The function Euler_inv (g, c, w, s, q), defined in (3.7), coincides with the contribution (3.6) if no $\psi$-classes are involved. Otherwise, Psi (, , c , w , s, q, a), defined in (3.8), corrects this discrepancy (see (5.3) for an example). The argument a in the definition of the function Psi is an array of integers representing the exponents of the $\psi$-classes.

After each loop of line 8 the value of

$$
\mathrm{P}(\mathrm{g}, \mathrm{c}, \mathrm{w}, \mathrm{s}, \mathrm{q}) * \frac{\operatorname{Euler} \_\operatorname{inv}(\mathrm{g}, \mathrm{c}, \mathrm{w}, \mathrm{s}, \mathrm{q})}{\operatorname{aut} * \operatorname{prod}(\mathrm{w})},
$$

which depends on $\mathbf{s}$, is added to ans. The value of ans after the completion of all the loops does not depend on $\mathbf{s}$.

Remark 4.1. As we said before, we consider aut as the order of the group of automorphisms of $\mathrm{g}$ preserving $\mathrm{c}$, and not those preserving the full decorated graph (that is, including $w$ and $q$ ) as in Eq. (3.2). This because we would have to deal with a much bigger database of decoreted graphs. The computation could have been faster, but with a high impact on user's hard-disk. However this does not change the final result since we run the loops for all possible weights and markings.

Remark 4.2. We use the Nemo package [FHHJ17] for rational number arithmetic instead of the much slower Julia's built-in Rational\{BigInt\}. We thank Jieao Song for suggesting us this improvement.

Remark 4.3. The current version of the package is 2.0.0. In the future, some parts of the code may be improved. We refer to the internal documentation of the package for an up-to-date explanation of its features. 


\section{Explicit Computations}

An implementation of the computational methods described in this paper is available in the Julia language. The implementation can be obtained from the repository https://github.com/mgemath/AtiyahBott.jl. To use our code, the user should first define the equivariant classes to be calculated. For example, the user might type

$P=$ Hypersurface (5);

The supported equavariant classes are listed in the file EquivariantClasses.jl (see also below for some explicit examples). After P is defined, one has to call the Atiyah-Bott formula by the command

AtiyahBottFormula (n, d, m, P);

This function will calculate $\mathrm{P}$ in the moduli space $\bar{M}_{0, m}\left(\mathbb{P}^{n}, d\right)$, as explained in the previous section. For multiple computations in the same moduli space, the variable $\mathrm{P}$ can be an array of equivariant classes. This way, the computation can be done simultaneously for several equivariant classes and this reduces the running time.

More detailed documentation of the package can be found in the github repository referred to above. Some examples with timing (in seconds) are given in the rest of the section. All computations were run on an Intel ${ }^{\circledR}$ Xeon ${ }^{\circledR}$ E-2244G CPU running at $3.80 \mathrm{GHz}$ with $64 \mathrm{~GB}$ of memory and 8 threads, using Version 2.0.0 of the package.

This program can support any polynomial in equivariant classes of vector bundles over $\bar{M}_{0, m}\left(\mathbb{P}^{n}, d\right)$, as long as the problem can be translated to a graph-theoretic computation, as in the examples below. In case the user needs to add more such classes, the authors are available for support.

\subsection{Projective spaces.}

5.1.1. Plane curves. We know that the invariant

$$
\int_{\bar{M}_{0,3 d-1}\left(\mathbb{P}^{2}, d\right)} \prod_{i=1}^{3 d-1} \mathrm{ev}_{i}^{*}(h)^{2}
$$

equals the number of rational plane curves of degree $d$ through $3 d-1$ general points. The computation of this invariant can be performed with our package as follows:

$\mathrm{d}=1$; \#for other values of $\mathrm{d}$, change this line

$P=01() \sim 2$;

AtiyahBottFormula (2,d,3*d-1,P);

The results with running times are listed in Table 5.1 This particular computation is not optimal since, as one can deduce from Example 3.2, the marks make the number of connected components of $\bar{M}_{0,3 d-1}\left(\mathbb{P}^{2}, d\right)^{T}$ much bigger than $\bar{M}_{0,0}\left(\mathbb{P}^{2}, d\right)^{T}$. Using Remark 2.1 (that is, the case $m=0$ ), we can drastically reduce the number of decorated graphs, speeding up the computation. Whenever possible, we will adopt this simplification in the forthcoming examples.

5.1.2. Contact curves. By the previous section, we know that the number of rational contact curves in $\mathbb{P}^{3}$ meeting $a$ general points and $b=2(d-a)+1$ general lines is

$$
\int_{\bar{M}_{0,0}\left(\mathbb{P}^{3}, d\right)} \delta_{*}\left(\mathrm{ev}_{1}^{*}\left(h^{3}\right)\right)^{a} \cdot \delta_{*}\left(\mathrm{ev}_{1}^{*}\left(h^{2}\right)\right)^{b} \cdot c_{\mathrm{top}}\left(\mathcal{E}_{d, 0}\right) .
$$


TABLE 5.1. Numbers of rational plane curves.

\begin{tabular}{c|c|c}
$d$ & value & runtime \\
\hline 1 & 1 & $0.00661 \mathrm{~s}$ \\
2 & 1 & $0.0259 \mathrm{~s}$ \\
3 & 12 & $13.9 \mathrm{~s}$
\end{tabular}

This invariant can be computed as follows:

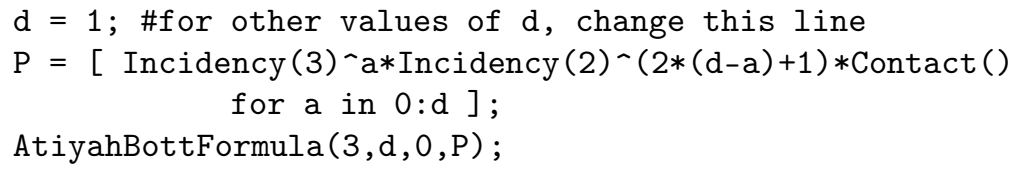

In Table 5.2 we list the values up to degree 8 . The values of the table for $a=0$ and $d \leq 4$ where computed in [LV11, Amo14]. All values for $d \leq 3$ where computed in Mur21.

TABLE 5.2. Numbers of rational contact space curves.

\begin{tabular}{|c|c|c|c|c|c|c|c|}
\hline$d$ & $\mathrm{a}$ & value & runtime & $d$ & $\mathrm{a}$ & value & runtime \\
\hline \multirow[t]{2}{*}{1} & 0 & 2 & $0.0006 \mathrm{~s}$ & 5 & 0 & 539504640 & \multirow[t]{6}{*}{$0.4715 \mathrm{~s}$} \\
\hline & 1 & 1 & & & 1 & 37318656 & \\
\hline \multirow[t]{3}{*}{2} & 0 & 40 & \multirow[t]{3}{*}{$0.003 \mathrm{~s}$} & & 2 & 2780160 & \\
\hline & 1 & 8 & & & 3 & 224896 & \\
\hline & 2 & 2 & & & 4 & 20000 & \\
\hline \multirow[t]{4}{*}{3} & 0 & 4160 & \multirow[t]{4}{*}{$0.049 \mathrm{~s}$} & & 5 & 2000 & \\
\hline & 1 & 512 & & 6 & 0 & 434591612928 & \multirow[t]{7}{*}{$2.299 \mathrm{~s}$} \\
\hline & 2 & 72 & & & 1 & 24641224704 & \\
\hline & 3 & 12 & & & 2 & 1484648448 & \\
\hline \multirow[t]{5}{*}{4} & 0 & 1089024 & \multirow[t]{5}{*}{$0.323 \mathrm{~s}$} & & 3 & 95545344 & \\
\hline & 1 & 96512 & & & 4 & 6614016 & \\
\hline & 2 & 9408 & & & 5 & 497664 & \\
\hline & 3 & 1024 & & & 6 & 41472 & \\
\hline & 4 & 128 & & \multirow[t]{9}{*}{8} & 0 & 858416911346565120 & \multirow[t]{9}{*}{$164.9 \mathrm{~s}$} \\
\hline \multirow[t]{8}{*}{7} & 0 & 518244677713920 & \multirow[t]{8}{*}{$16.02 \mathrm{~s}$} & & 1 & 35755655304314880 & \\
\hline & 1 & 24890475282432 & & & 2 & 1557262876803072 & \\
\hline & 2 & 1258547527680 & & & 3 & 71102066589696 & \\
\hline & 3 & 67234406400 & & & 4 & 3414199762944 & \\
\hline & 4 & 3812150272 & & & 5 & 173116293120 & \\
\hline & 5 & 230814720 & & & 6 & 9319309312 & \\
\hline & 6 & 15059072 & & & 7 & 536870912 & \\
\hline & 7 & 1075648 & & & 8 & 33554432 & \\
\hline
\end{tabular}

5.1.3. Tangent curves. Let $Y$ be a smooth plane curve of degree $z$. The number of lines tangent to $Y$ and passing through a general point is classically known to be $z(z-1)$. This can be proved using Grassmannians (see [EH16, Section 3.6.2]). Let us prove it using the moduli space of stable curves. Following Gat02, Definition 1.1], we denote by $\bar{M}_{(2,0)}^{Y}\left(\mathbb{P}^{2}, 1\right)$ the moduli space of stable maps in $\bar{M}_{0,2}\left(\mathbb{P}^{2}, 1\right)$ tangent to $Y$ at the first marked points. If $x \in \mathbb{P}^{2}$ is a general point, we have 


$$
\begin{aligned}
z(z-1) & =\int_{\left.\left[\bar{M}_{(2,0)}^{Y}\left(\mathbb{P}^{2}, 1\right)\right]\right]^{\mathrm{virt}}} \mathrm{ev}_{2}^{*}(x) \\
& =\int_{\bar{M}_{0,2}\left(\mathbb{P}^{2}, 1\right)} c_{\mathrm{top}}\left(\mathrm{J}^{1}\left(\operatorname{ev}_{1}^{*} \mathcal{O}_{\mathbb{P}^{2}}(z)\right)\right) \cdot \mathrm{ev}_{2}^{*}\left(h^{2}\right)
\end{aligned}
$$

Equation 5.1 is an application of Kleiman-Bertini Kle74]: it is well-know that $\bar{M}_{(2,0)}^{Y}\left(\mathbb{P}^{2}, 1\right)$ is of the expected dimension (in particular, its virtual fundamental class equals its usual fundamental class), thus for a general $x$ the subspaces $\bar{M}_{(2,0)}^{Y}\left(\mathbb{P}^{2}, 1\right)$ and $e_{2}^{-1}(x)$ meet transversely in a zero cycle. Equation $[5.2$ is deduced from [Gat02, Theorem 2.6].

Using (2.2) and Remark 2.2 with $p=1$ :

$$
0 \rightarrow \mathrm{ev}_{1}^{*} \mathcal{O}_{\mathbb{P}^{n}}(z) \otimes \mathbb{L}_{1} \rightarrow \mathrm{J}^{1}\left(\mathrm{ev}_{1}^{*} \mathcal{O}_{\mathbb{P}^{n}}(z)\right) \rightarrow \mathrm{ev}_{1}^{*} \mathcal{O}_{\mathbb{P}^{n}}(z) \rightarrow 0,
$$

the integral in Equation (5.2) can be expanded as

$$
\int_{\bar{M}_{0,2}\left(\mathbb{P}^{2}, 1\right)} z \operatorname{ev}_{1}^{*}(h) \cdot\left(z \operatorname{ev}_{1}^{*}(h)+\psi_{1} \psi_{2}^{0}\right) \cdot \operatorname{ev}_{2}^{*}\left(h^{2}\right)
$$

It can be computed with the following instructions:

$$
\begin{aligned}
& z=1 \text {; \#for other values of } z \text {, change this line } \\
& P=z * 01 \_i(1) *\left(z * 01 \_i(1)+P \operatorname{si}([1,0])\right) * 01 \_i(2)-2 ; \\
& \text { AtiyahBottFormula }(2,1,2, P) ;
\end{aligned}
$$

The number of flex tangents of a smooth plane curve of degree $z$ is exactly

$$
3 z(z-2)=\int_{\bar{M}_{0,1}\left(\mathbb{P}^{2}, 1\right)} c_{\mathrm{top}}\left(\mathrm{J}^{2}\left(\mathrm{ev}_{1}^{*} \mathcal{O}_{\mathbb{P}^{2}}(z)\right)\right),
$$

see [EH16, Section 11.3]. We can compute it either by expanding the Euler class of the jet bundle, or by using the function Jet provided by the package:

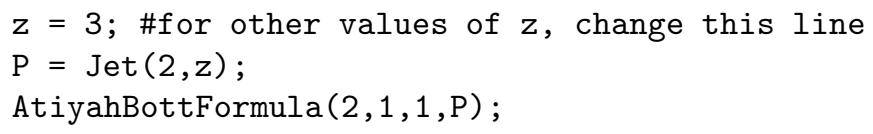

Other interesting invariants can be deduced using the jet bundle. For example,

$$
\int_{\bar{M}_{0,1}\left(\mathbb{P}^{n}, d\right)} \operatorname{ev}_{1}^{*}\left(h^{n-1}\right) \cdot c_{\mathrm{top}}\left(\mathrm{J}^{(n+1) d-2}\left(\operatorname{ev}_{1}^{*} \mathcal{O}_{\mathbb{P}^{m}}(1)\right)\right)
$$

is an invariant related to the osculating curves of a hypersurface in $\mathbb{P}^{n}$, see Mur21] and references therein. It can be calculated with

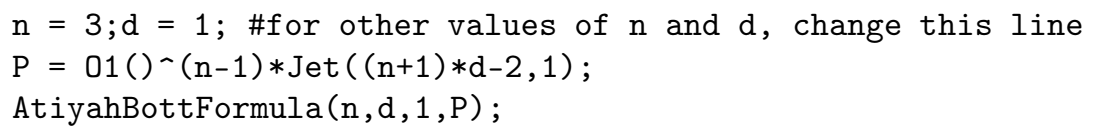




\subsection{Calabi-Yau Threefolds.}

5.2.1. Quintic. Let $X \subset \mathbb{P}^{4}$ be a general quintic hypersurface. We may define the Gromov-Witten invariants as

$$
N_{d}:=\int_{\bar{M}_{0,0}\left(\mathbb{P}^{4}, d\right)} c_{\mathrm{top}}\left(\delta_{*}\left(\operatorname{ev}_{1}^{*} \mathcal{O}_{\mathbb{P}^{4}}(5)\right)\right),
$$

and recursively the instanton numbers $n_{d}$ by the formula

$$
N_{d}=\sum_{k \mid d} n_{\frac{d}{k}} k^{-3} \text {. }
$$

It was proved, for $d \leq 9$, by [JK96] that $n_{d}$ equals the number of rational curves in $X$ of degree $d$. All values of $n_{d}$ are predicted by Mirror Symmetry (see CdlOGP92]). In Table 5.3 we list the values of $N_{d}$ up to $d=9$. We used the following code.

$\mathrm{d}=1$; \#for other values of $\mathrm{d}$, change this line

$P=$ Hypersurface (5);

AtiyahBottFormula (4, d, 0,P);

Kontsevich [Kon95] reported that the computation for $N_{4}$ took 5 minutes on a Sun computer in 1995 - now we can do it in a fraction of a second. In order to get the real

TABLE 5.3. GW invariants of a quintic threefold.

\begin{tabular}{c|c|c}
$d$ & value & runtime \\
\hline 1 & 2875 & $0.0004 \mathrm{~s}$ \\
2 & $4876875 / 8$ & $0.00145 \mathrm{~s}$ \\
3 & $8564575000 / 27$ & $0.004 \mathrm{~s}$ \\
4 & $15517926796875 / 64$ & $0.028 \mathrm{~s}$ \\
5 & 229305888887648 & $0.243 \mathrm{~s}$ \\
6 & 248249742157695375 & $2.01 \mathrm{~s}$ \\
7 & $101216230345800061125625 / 343$ & $33.1 \mathrm{~s}$ \\
8 & $192323666400003538944396875 / 512$ & $377 \mathrm{~s}$ \\
9 & $367299732093982242625847031250 / 729$ & $4239 \mathrm{~s}$
\end{tabular}

number of rational curves, we can apply Manin's formula (2.5) by removing from $N_{d}$ the contribution of the covers of the curves of lower degree. Such a contribution can be obtained by:

$d=1$; \#for other values of $d$, change this line

$P=R 1(1)^{\sim} 2$;

AtiyahBottFormula (1, d, 0,P);

These numbers were computed using these methods up to degree 6 by [Hie16]. Moreover, they have been computed using the original physical methods for large $d$, see [OEIS, A060041] and reference therein.

5.2.2. Other threefolds. By the adjunction formula, it can easily be seen that there are four other possibilities of complete intersection Calabi-Yau threefolds other than the quintic hypersurface in $\mathbb{P}^{4}$. These are complete intersections of multidegree $(3,3)$ and $(4,2)$ in $\mathbb{P}^{5},(3,2,2)$ in $\mathbb{P}^{6}$ and $(2,2,2,2)$ in $\mathbb{P}^{7}$. We computed some of the Gromov-Witten numbers of these varieties in Table 5.4. Our results agree with [LT93. See also AK91, Ber08] and references therein. 
TABLE 5.4. GW invariants of Calabi-Yau threefolds.

\begin{tabular}{c|c|c||c|c|c}
\multicolumn{3}{c||}{$(3,3)$} & \multicolumn{3}{c}{$(4,2)$} \\
\hline$d$ & value & runtime & $d$ & value & runtime \\
\hline 1 & 1053 & $0.0004 \mathrm{~s}$ & 1 & 1280 & $0.0007 \mathrm{~s}$ \\
2 & $423549 / 8$ & $0.0023 \mathrm{~s}$ & 2 & 92448 & $0.0025 \mathrm{~s}$ \\
3 & 6424365 & $0.0103 \mathrm{~s}$ & 3 & $422690816 / 27$ & $0.0112 \mathrm{~s}$ \\
4 & $72925120125 / 64$ & $0.1019 \mathrm{~s}$ & 4 & 3883914084 & $0.8888 \mathrm{~s}$ \\
\hline \multicolumn{3}{c|}{$(3,2,2)$} & \multicolumn{3}{|c}{$(2,2,2,2)$} \\
\hline$d$ & value & runtime & $d$ & value & runtime \\
\hline 1 & 720 & $0.0005 \mathrm{~s}$ & 1 & 512 & $0.0009 \mathrm{~s}$ \\
2 & 22518 & $0.0037 \mathrm{~s}$ & 2 & 9792 & $0.0056 \mathrm{~s}$ \\
3 & $4834592 / 3$ & $0.0252 \mathrm{~s}$ & 3 & $11239424 / 27$ & $0.0455 \mathrm{~s}$ \\
4 & $672808059 / 4$ & $0.3669 \mathrm{~s}$ & 4 & 25705160 & $0.7849 \mathrm{~s}$
\end{tabular}

\subsection{Fano varieties.}

5.3.1. Cubic surface. Let $X \subset \mathbb{P}^{3}$ be a general cubic surface. Complete recursion formulae for the enumerative numbers of $X$ are known from DFI95, where all of them are listed up to degree 6 . It is known that the number

$$
\int_{\bar{M}_{0, d-1}\left(\mathbb{P}^{3}, d\right)} \operatorname{ev}_{1}^{*}\left(h^{2}\right) \cdots \mathrm{ev}_{d-1}^{*}\left(h^{2}\right) \cdot c_{\mathrm{top}}\left(\delta_{*}\left(\operatorname{ev}_{d}^{*} \mathcal{O}_{\mathbb{P}^{3}}(3)\right)\right)
$$

is enumerative. Moreover, a general line in $\mathbb{P}^{3}$ meets $X$ at three points, so if we want to compute the number of curves in $X$ through $d-1$ general points, we need to multiply the above display by $3^{1-d}$. In Table 5.5 we listed the number of rational curves of degree $d$ on $X$ meeting $d-1$ general points up to degree 8. Our results are all in agreement with existing results.

TABLE 5.5. Numbers curves in a cubic surface.

\begin{tabular}{c|c|c||c|c|c}
$d$ & value & runtime & $d$ & value & runtime \\
\hline 1 & 27 & $0.0003 \mathrm{~s}$ & 5 & 5616 & $0.0538 \mathrm{~s}$ \\
2 & 27 & $0.0008 \mathrm{~s}$ & 6 & 82944 & $0.287 \mathrm{~s}$ \\
3 & 84 & $0.0019 \mathrm{~s}$ & 7 & 1608768 & $3.9125 \mathrm{~s}$ \\
4 & 540 & $0.0082 \mathrm{~s}$ & 8 & 38928384 & $36 \mathrm{~s}$
\end{tabular}

5.3.2. Quadric fourfold. Complete recursion formulae for the quadric surfaces and threefolds are in [DFI95, FP97]. In Table 5.6 we listed the values of the invariant

$$
\int_{\bar{M}_{0,0}\left(\mathbb{P}^{5}, d\right)} \delta_{*}\left(\mathrm{ev}_{1}^{*}\left(h^{4}\right)\right)^{a} \cdot \delta_{*}\left(\mathrm{ev}_{1}^{*}\left(h^{3}\right)\right)^{b} \cdot \delta_{*}\left(\mathrm{ev}_{1}^{*}\left(h^{2}\right)\right)^{c} \cdot c_{\mathrm{top}}\left(\delta_{*}\left(\operatorname{ev}_{1}^{*} \mathcal{O}_{\mathbb{P}^{5}}(2)\right)\right),
$$

where $3 a+2 b+c=4 d+1$ for $d=1$ and $d=2$. Both tables required far less than a second of CPU time. 
TABLE 5.6. Numbers of lines and conics in a quadric fourfold.

\begin{tabular}{|c|c|c|c|c|}
\hline & $(a, b, c)$ & value & $(a, b, c)$ & value \\
\hline & $(0,0,5)$ & 20 & $(0,2,1)$ & 8 \\
\hline & $(0,1,3)$ & 12 & $(1,1,0)$ & 4 \\
\hline & $(1,0,2)$ & 4 & & \\
\hline$(a, b, c)$ & \multicolumn{2}{|c|}{ value } & $(a, b, c)$ & value \\
\hline$(0,0,11)$ & \multicolumn{2}{|c|}{338878012462176} & $(0,4,3)$ & 2868685444352 \\
\hline$(0,1,9)$ & \multicolumn{2}{|c|}{101436360044288} & $(0,5,1)$ & 866614157376 \\
\hline$(0,2,7)$ & \multicolumn{2}{|c|}{30581975310256} & $(1,0,8)$ & 20679736185808 \\
\hline$(0,3,5)$ & \multicolumn{2}{|c|}{9330647718128} & $(1,1,6)$ & 6274105265472 \\
\hline$(1,2,4)$ & \multicolumn{2}{|c|}{1958615970176} & $(2,1,3)$ & 422160042016 \\
\hline$(1,3,2)$ & \multicolumn{2}{|c|}{617652337904} & $(2,2,1)$ & 139428589504 \\
\hline$(1,4,0)$ & \multicolumn{2}{|c|}{185149566080} & $(3,0,2)$ & 103721819712 \\
\hline$(2,0,5)$ & \multicolumn{2}{|c|}{1260340192768} & $(3,1,0)$ & 35140354848 \\
\hline
\end{tabular}

\section{REFERENCES}

[AB84] M. F. Atiyah and R. Bott, The moment map and equivariant cohomology, Topology 23 (1984), no. 1, 1-28. MR 721448

[AK91] Alberto Albano and Sheldon Katz, van Geemen's families of lines on special quintic threefolds, Manuscripta Math. 70 (1991), no. 2, 183-188. MR 1085631

[AM93] Paul S. Aspinwall and David R. Morrison, Topological field theory and rational curves, Comm. Math. Phys. 151 (1993), no. 2, 245-262. MR 1204770

[Amo14] Éden Amorim, Curvas de contato no espaço projetivo, Ph.D. thesis, Universidade Federal de Minas Gerais, Belo Horizonte, Brazil, 2014, In English: Contact curves in the projective space, arXiv:1907.03973.

[BEKS17] Jeff Bezanson, Alan Edelman, Stefan Karpinski, and Viral B. Shah, Julia: a fresh approach to numerical computing, SIAM Rev. 59 (2017), no. 1, 65-98. MR 3605826

[Ber08] M. Bernardara, Calabi-Yau complete intersections with infinitely many lines, Rend. Semin. Mat. Univ. Politec. Torino 66 (2008), no. 2, 87-97. MR 2455452

[Bot67] Raoul Bott, A residue formula for holomorphic vector-fields, J. Differential Geometry 1 (1967), 311-330. MR 232405

[CdlOGP92] Philip Candelas, Xenia C. de la Ossa, Paul S. Green, and Linda Parkes, A pair of Calabi-Yau manifolds as an exactly soluble superconformal theory, Essays on mirror manifolds, Int. Press, Hong Kong, 1992, pp. 31-95. MR 1191420

[CK99] David A. Cox and Sheldon Katz, Mirror symmetry and algebraic geometry, Mathematical Surveys and Monographs, vol. 68, American Mathematical Society, Providence, RI, 1999. MR 1677117

[DFI95] P. Di Francesco and C. Itzykson, Quantum intersection rings, The moduli space of curves (Texel Island, 1994), Progr. Math., vol. 129, Birkhäuser Boston, Boston, MA, 1995, pp. 81-148. MR 1363054

[EH16] David Eisenbud and Joe Harris, 3264 and all that-a second course in algebraic geometry, Cambridge University Press, Cambridge, 2016. MR 3617981

[ES96] Geir Ellingsrud and Stein Arild Strømme, Bott's formula and enumerative geometry, J. Amer. Math. Soc. 9 (1996), no. 1, 175-193. MR 1317230

$\left[\mathrm{FBS}^{+} 21\right]$ James Fairbanks, Mathieu Besançon, Simon Schölly, Júlio Hoffiman, Nick Eubank, and Stefan Karpinski, JuliaGraphs/Graphs.jl: an optimized graphs package for the Julia programming language, 2021.

[FHHJ17] Claus Fieker, William Hart, Tommy Hofmann, and Fredrik Johansson, Nemo/Hecke: computer algebra and number theory packages for the Julia programming language, ISSAC'17-Proceedings of the 2017 ACM International Symposium on Symbolic and Algebraic Computation, ACM, New York, 2017, pp. 157-164. MR 3703682 
[FP97] W. Fulton and R. Pandharipande, Notes on stable maps and quantum cohomology, Algebraic geometry-Santa Cruz 1995, Proc. Sympos. Pure Math., vol. 62, Amer. Math. Soc., Providence, RI, 1997, pp. 45-96. MR 1492534

[Gat02] Andreas Gathmann, Absolute and relative Gromov-Witten invariants of very ample hypersurfaces, Duke Math. J. 115 (2002), no. 2, 171-203. MR 1944571

[GP99] T. Graber and R. Pandharipande, Localization of virtual classes, Invent. Math. 135 (1999), no. 2, 487-518. MR 1666787

[Hie16] Dang Tuan Hiep, Rational curves on Calabi-Yau threefolds: verifying mirror symmetry predictions, J. Symbolic Comput. 76 (2016), 65-83. MR 3461259

[JK96] Trygve Johnsen and Steven L. Kleiman, Rational curves of degree at most 9 on a general quintic threefold, Comm. Algebra 24 (1996), no. 8, 2721-2753. MR 1393281

[Kle74] Steven L. Kleiman, The transversality of a general translate, Compositio Math. 28 (1974), 287-297. MR 360616

[Kob59] Shoshichi Kobayashi, Remarks on complex contact manifolds, Proc. Amer. Math. Soc. 10 (1959), 164-167. MR 111061

[Kon92] Maxim Kontsevich, Intersection theory on the moduli space of curves and the matrix Airy function, Comm. Math. Phys. 147 (1992), no. 1, 1-23. MR 1171758

[Kon95] _ Enumeration of rational curves via torus actions, The moduli space of curves (Texel Island, 1994), Progr. Math., vol. 129, Birkhäuser Boston, Boston, MA, 1995, pp. 335-368. MR 1363062

[LP04] Y.-P. Lee and Rahul Pandharipande, Frobenius manifolds, Gromov-Witten theory, and Virasoro constraints, 2004, available at www.math.utah.edu/ yplee/research.

[LS17] Chiu-Chu Melissa Liu and Artan Sheshmani, Equivariant Gromov-Witten invariants of algebraic GKM manifolds, SIGMA Symmetry Integrability Geom. Methods Appl. 13 (2017), Paper No. 048, 21. MR 3667222

[LT93] A. Libgober and J. Teitelbaum, Lines on Calabi-Yau complete intersections, mirror symmetry, and Picard-Fuchs equations, Internat. Math. Res. Notices (1993), no. 1, 29-39. MR 1201748

[LV11] D. Levcovitz and I. Vainsencher, Symplectic enumeration, Bull. Braz. Math. Soc. (N.S.) 42 (2011), no. 3, 347-358. MR 2833807

[Man95] Yu. I. Manin, Generating functions in algebraic geometry and sums over trees, The moduli space of curves (Texel Island, 1994), Progr. Math., vol. 129, Birkhäuser Boston, Boston, MA, 1995, pp. 401-417. MR 1363064

[Mur20] Giosuè Muratore, Enumeration of rational contact curves via torus actions, 2020, Michigan Math. J. (to appear).

[Mur21] Giosuè Muratore, A recursive formula for osculating curves, Ark. Mat. 59 (2021), no. 1, 195-211. MR 4256011

[OEIS] OEIS Foundation Inc, The On-Line Encyclopedia of Integer Sequences, 2022, http://oeis.org.

[Pan13] R. Pandharipande, Convex rationally connected varieties, Proc. Amer. Math. Soc. 141 (2013), no. 5, 1539-1543. MR 3020841

[Voi96] Claire Voisin, A mathematical proof of a formula of Aspinwall and Morrison, Compositio Math. 104 (1996), no. 2, 135-151. MR 1421397

[Wit91] Edward Witten, Two-dimensional gravity and intersection theory on moduli space, Surveys in differential geometry (Cambridge, MA, 1990), Lehigh Univ., Bethlehem, PA, 1991, pp. 243-310. MR 1144529

[Ye94] Yun-Gang Ye, A note on complex projective threefolds admitting holomorphic contact structures, Invent. Math. 115 (1994), no. 2, 311-314. MR 1258907

Department of Mathematics, Universidade Federal de Minas Gerais, Belo Horizonte, MG, Brazil.

Email address: muratore.g.e@gmail.com, csaba.schneider@gmail.com

$U R L:$ https://sites.google.com/view/giosue-muratore 